\title{
Social Knowledge and Attitude toward Over-the-Counter Drug Use
}

\author{
Malak Khalifeh ${ }^{1,2, *}$, Nicholas Moore ${ }^{1}$, Pascale Salameh ${ }^{2}$ \\ ${ }^{1}$ INSERM U 657 - Pharmaco-épidémiologie et évaluation de l'impact des produits de santé sur les populations, \\ University Bordeaux Segalen, Bordeaux, France \\ ${ }^{2}$ Clinical \& Epidemiological Research Laboratory, Faculty of Pharmacy, Lebanese University, Beirut, Lebanon \\ *Corresponding author: malak.k9@hotmail.com
}

\begin{abstract}
Introduction: With the increasing range of potent medicines available for sale 'over-the-counter' (OTC) in community pharmacies and the absence of data in Lebanon related to OTC abuse, this study sought to find opinions of the public regarding OTC medicines generally and explore views around potential misuse and abuse. Method: A cross sectional study was conducted in a community-based pharmacy setting in Lebanon. A structured random interview was used to patients visiting community pharmacy seeking for OTC drugs. Baseline characteristics and reason for self-medication were collected. The questionnaire consists of sections about sources and reasons of self-medication, attitudes towards community pharmacy and patient contact with pharmacies, attitudes towards the use of OTC medicines; views on OTC medicines' use in terms of safety, potency and effectiveness. Result: Overall this study revealed the knowledge and attitude of general public toward OTC use in Lebanon. Self-presentation on previous experience of the same medication or through pharmacist advice. Almost half requested OTC medications more than twice per year. This show the important role of pharmacist in guiding the patients on the correct OTC use. Around 60\% agreed that some non-prescription medicines may cause dependency or addiction if taken for a long period of time. Conclusion: The findings of this research should form the basis for future interventional plans to maximize benefits and minimize risks of self-medication practice. Educational programs to population, pharmacists and health care providers should be implemented to limit the potential misuse/abuse of these medicines.
\end{abstract}

Keywords: self-medication, OTC, Lebanon, public views, knowledge, attitude

Cite This Article: Malak Khalifeh, Nicholas Moore, and Pascale Salameh, "Social Knowledge and Attitude toward Over-the-Counter Drug Use." American Journal of Clinical Medicine Research, vol. 6, no. 2 (2018): 35-40. doi: 10.12691/ajcmr-6-2-3.

\section{Introduction}

One of the quiet revolution occurring in our pharmaceutical industry is the transformation of prescription medications to over the counter medications ((OTC) referred to non-prescription medications or self-medications). Although OTC drugs are believed to be safe and effective, indeed they are not. They mask the underlying disease and may cause several adverse effects [1], with increased risks of interactions and adverse reactions and of self-treatment being undertaken when medical aid should have been sought. [2,3] There is also the potential for misuse and abuse of such products [4].

The misuse of codeine products is an emerging public health challenge in many countries around the world, in part because such products are present in a range of OTC medicines regularly dispensed without prescription to the public[5]. The greatest proportion of evidence on codeine misuse and dependence is from the United States (US) where codeine based products are not available over the counter, with smaller proportions (but not directly comparable) of studies on OTC codeine use and misuse conducted in the United Kingdom (UK) and Australia.

A recent review on the changing patterns of codeine based product use was conducted by Reed et al., (2011) and reported on levels of public awareness of misuse potential, and pharmacists' continued concerns for misuse. [6] Reed et al., (2011) observed the need for further investigation, as OTC codeine based drugs become available, either as sold by and under supervision of a pharmacist or sold off the shelf from any outlet. [6] A clearer understanding of the general public's awareness on the potential risks of codeine use, misuse and dependence, and impact of pharmacy controls of codeine is needed [6,7]. Although effects are milder than heroin, abuse potential remains of concern, with physical dependence occurring with regular use over a short period of time. [8]

According to the EMCDDA Annual Report in 2010, the misuse of prescribed and OTC medication, and the sale of counterfeit medicines sold for therapeutic and nontherapeutic use is now under consideration within the European Pharmaco-vigilance system, which operates under the responsibility of the European Medicines Agency, and collaborates with the EMCDDA on drug misuse issues. The report underscores the need for timely responses from pharmaceutical and drug control regulatory 
frameworks [9]. Greater efforts to increase user awareness and self-monitoring of OTC product use are emphasized in a variety of studies $[10,11]$. Hughes (2002) emphasized how self-medication using OTC products is beneficial to all facets of society, but warned of the need to monitor certain key populations and products $[3,8]$.

With the increasing range of potent medicines available for sale 'over-the-counter' (OTC) in community pharmacies and the absence of data in Lebanon related to OTC abuse, this study sought to find opinions of the public regarding OTC medicines generally and explore views around potential misuse and abuse.

\section{Method}

\subsection{Study Design}

A cross sectional study was conducted in a communitybased pharmacy setting in Lebanon. Data was collected over 1 year (September 2015 to September2016) from 50 community pharmacies CPs distributed in six different districts in Lebanon: Beirut, South Lebanon, Nabatiyeh, Mount Lebanon, Bekaa, and North. Data was collected using a structured random interview conducted by study researchers who had been briefed about the study's aims and methods. Consecutive customers arriving at community pharmacies seeking OTC medications were interviewed. The interview was based on a well-structured questionnaire, which had been pre-tested on a small pilot scale and subsequently modified to ensure that the data would provide reliable information.

The patients were informed about the objective of the study and were asked to give an oral consent before they start of the procedure. Only those who were interested and who give their voluntary informed oral consent to participate in the study were enrolled. Also, they were informed that the information will not be given to anybody. Patients were assured of their anonymity and confidentiality of responses. The Lebanese University, Faculty of Pharmacy Internal Review Board waived the need for oral informed consent.

\subsection{Study Population}

Eligible participants were recruited randomly from consumers presenting at community-based pharmacies in Lebanon after they have purchased OTC drugs liable for abuse. The patients included were from both genders, aged 16 years and older come for purchasing non-prescription medication. Exclusion criteria was children of younger than 16 years old and patients requesting OTC drugs for their relatives.

A sample size was calculated according to Epi info assuming a type I error of $5 \%$ and a study power of $80 \%$ and CI 95\%. There is no data on the prevalence of OTC use in Lebanon. However, the prevalence of self-medication in Jordan is available and was around $42 \%$ [12]. The minimal sample size necessary to show a twofold increase in the risk of exposure to OTC medication consists of 442 subjects.

\subsection{Data Collection}

The questionnaire was obtained after wide review of literature [13]. It was divided into five sections: socio- demographic data (age, sex, occupation, educational and marital status, monthly income, medical insurance, and the presence of a care provider at home), lifestyle data (smoking status, alcohol status, and involvement in sport activities). It also consisted of attitudes towards the use of OTC medicines; views on OTC medicines' use in terms of safety, potency and effectiveness; and an exploration of knowledge and opinion on the inappropriate use of OTC medicines, misuse and abuse.

\subsection{Data Analysis}

All data were coded and entered and analyzed using the SPSS version19. Frequencies and percentages of sociodemographic characteristics, reasons and sources for non-prescription use, classes of non-prescription medications, knowledge and attitude practices were calculated and presented. An appropriate bivariate analysis was done for every explanatory variable: A Chi-2 test was done for dichotomous, ordinal, and nominal variables. T-test or ANOVA was done for continuous variables. Every variable with a p-value of less than 0.2 in the bivariate analyses was entered into the multiple regressions.

\section{Results}

\subsection{Baseline Characteristics}

Data from a total of 462 participants were recorded. Among participants, $375(81.2 \%)$ requested for non-prescription medicines without prescription while 87 (18.8\%) had a medical prescription. The majority being between 16 and 50 years of age $(88.7 \%)$, while $11.3 \%$ were $>51$ years of age. A high percentage had a university degree $(56.3 \%)$, while $14.9 \%$ had secondary education, $28.8 \%$ had intermediate and elementary education. (see Table 1).

\subsection{Drug Utilization Patterns}

Self-medication practice was mainly self-presented by patients themselves based on previous experience with the same medication $(36 \%)$ or pharmacist advice $(30.1 \%)$. Of the self-medicated drugs, $13.3 \%$ were based on advice from a friend and/or family member and $19.5 \%$ based on physician advice. (Figure 1).

The main reason reported by patients for self-medication was previous successful use of OTC drugs liable for abuse $(53.4 \%)$, followed by saving time $(25.7 \%)$, or fear of the disease to become worse $(11.5 \%)$. The remaining reasons were saving the cost for the physician visit (18\%) and ease access of OTC drugs liable for abuse from community pharmacies (10.6\%) (Figure 2).

\subsection{Views of the Public on the Safety, Potency and Effectiveness of OTC Drugs}

Respondents' views on safety, potency, effectiveness and liability for abuse and dependence are presented in Table 2. $40.7 \%$ agreed that prescription-only-medicines should be deregulated to OTC status. Females $\left(\mathrm{X}^{2}=8.87\right.$, $\mathrm{df}=2, \mathrm{p}=.012)$ and younger participants $\left(\mathrm{X}^{2}=24.5, \mathrm{df}=4\right.$, $\mathrm{p}<.001)$ more agreed that prescription-only-medicines 
should be deregulated to OTC status. $44.7 \%$ started OTC medicines at the first sign of illness while $26.1 \%$ use OTC medicines only if the illness is quite severe. $22.9 \%$ thought that OTC medicines are totally safe while $51.2 \%$ believed that OTC medicines can have dangerous side effects. $55.8 \%$ believed that they can be as serious as that of prescription medicines. Around half believed that non-prescription medicines can sometimes mask serious health problems and interfere with the natural healing process of the body. $59.3 \%$ believed that with continual use, some non-prescription medicines lose their effectiveness. $61.4 \%$ believed that some non-prescription medicines may cause dependency or addiction if taken for a long period of time. Females and younger participants $(p<.001)$ believed that some non-prescription medicines may cause dependency or addiction.

Table 1. Sociodemographic characteristics of study participants

\begin{tabular}{|c|c|}
\hline & $\begin{array}{l}\text { Total participants } \\
\text { N=462 (100\%) }\end{array}$ \\
\hline With prescription & $87(18.8 \%)$ \\
\hline Without prescription & $375(81.2 \%)$ \\
\hline \multicolumn{2}{|l|}{ Gender } \\
\hline Male & $129(27.9 \%)$ \\
\hline Female & $333(72.1 \%)$ \\
\hline \multicolumn{2}{|l|}{ Age group } \\
\hline $16-25$ & $215(46.5 \%)$ \\
\hline 25-50 & $195(42.2 \%)$ \\
\hline$>\mathbf{5 0}$ & $52(11.3 \%)$ \\
\hline \multicolumn{2}{|l|}{ Educational level } \\
\hline Primary and less & $133(28.8 \%)$ \\
\hline Secondary & $69(14.9 \%)$ \\
\hline University & $260(56.3 \%)$ \\
\hline \multicolumn{2}{|l|}{ Marital status } \\
\hline Single & $242(52.4 \%)$ \\
\hline Married & $220(47.6 \%)$ \\
\hline \multicolumn{2}{|l|}{ Currently working } \\
\hline No & $304(65.8 \%)$ \\
\hline Yes & $158(34.2 \%)$ \\
\hline \multicolumn{2}{|l|}{ Family income (LL) } \\
\hline$<2000000$ & $128(27.7 \%)$ \\
\hline$>2000000$ & $36(7.8 \%)$ \\
\hline No response & $298(64.5 \%)$ \\
\hline \multicolumn{2}{|c|}{ Presence of comorbidities } \\
\hline Yes & $98(21.2 \%)$ \\
\hline No & $363(78.6 \%)$ \\
\hline
\end{tabular}

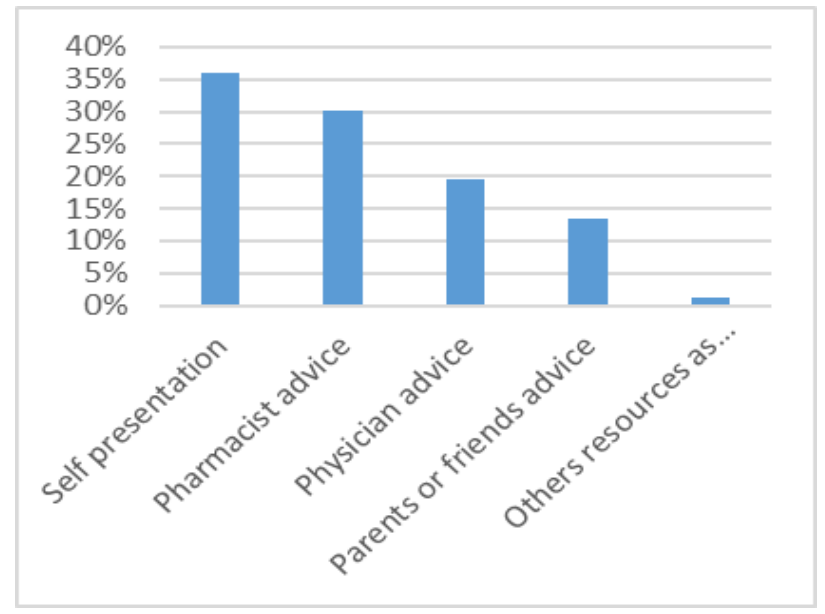

Figure 1. Sources of self-medication among participants requesting OTC drugs liable for abuse

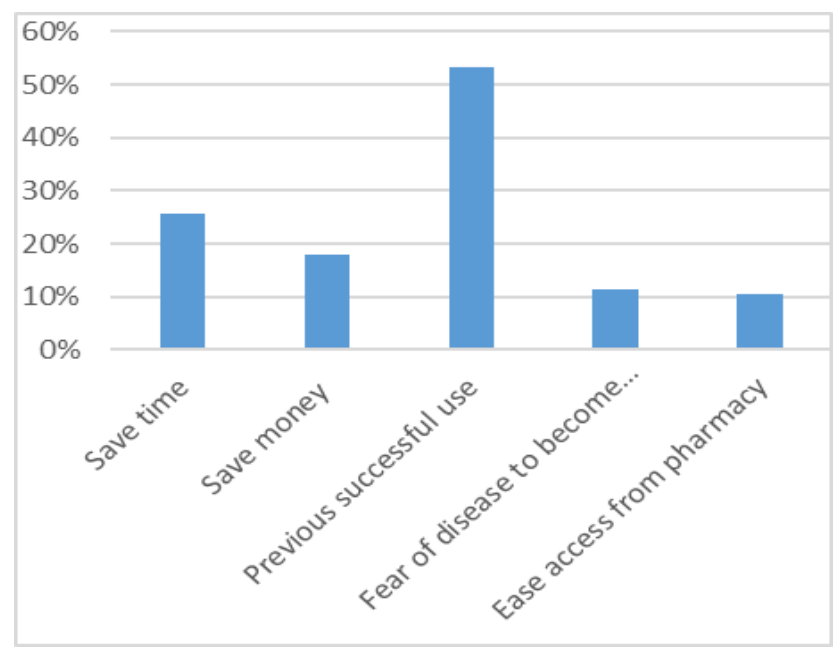

Figure 2. Reasons for self-medication among participants requesting OTC drugs liable for abuse

$43.4 \%$ reported that they always followed the directions on the OTC drug package, while $31.2 \%$ said they often followed the directions. $25.3 \%$ didn't follow the instructions of the label. Females $\left(X^{2}=13.9, \mathrm{df}=2, \mathrm{p}<.001\right)$ and younger participants $\left(\mathrm{X}^{2}=10.8, \mathrm{df}=4, \mathrm{p}=.029\right)$ were more likely to follow the directions on the packet. $89.7 \%$ had home pharmacies where females were more likely to have pharmacies at home $\left(\mathrm{X}^{2}=7.74, \mathrm{df}=1, \mathrm{p}=.005\right) .92 .8 \%$ checked the expiry use before use. $41.2 \%$ visited the pharmacy more than twice per year. (Table 3 )

Table 2. Views of the public on the Safety, potency and effectiveness of OTC drugs

\begin{tabular}{|c|c|c|c|}
\hline Total respondents $(n=462)$ & Agree & Disagree & Uncertain \\
\hline More POM drugs should be deregulated to OTC status & $186(40.7 \%)$ & $181(39.6 \%)$ & $90(19.7 \%)$ \\
\hline I reach for OTC medicines at the first sign of illness & $205(44.7 \%)$ & $185(40.3 \%)$ & $69(15.0 \%)$ \\
\hline Non-prescription medicines are totally safe to use & $105(22.9 \%)$ & $192(41.8 \%)$ & $162(35.3 \%)$ \\
\hline Non-prescription medicines can have dangerous side-effects & $234(51.2 \%)$ & $83(18.2 \%)$ & $140(30.6 \%)$ \\
\hline $\begin{array}{l}\text { The effect of incorrect use of non-prescription medicines can be as serious as that of } \\
\text { prescription medicines }\end{array}$ & $258(55.8 \%)$ & $98(21.5 \%)$ & $100(21.9 \%)$ \\
\hline Non-prescription medicines can sometimes mask serious health problems & $230(50.3 \%)$ & $106(23.2 \%)$ & $121(26.5 \%)$ \\
\hline Some non-prescription medicines interfere with the natural healing process of the body & $240(52.5 \%)$ & $84(18.4 \%)$ & $133(29.1 \%)$ \\
\hline With continual use, some non-prescription medicines lose their effectiveness & $271(59.3 \%)$ & $75(16.4 \%)$ & $111(24.3 \%)$ \\
\hline $\begin{array}{l}\text { Some non-prescription medicines may cause dependency or addiction if taken for a long } \\
\text { period of time. }\end{array}$ & $282(61.4 \%)$ & $82(17.9 \%)$ & $95(20.7 \%)$ \\
\hline
\end{tabular}


Table 3. General attitude toward OTC use

\begin{tabular}{|c|c|c|}
\hline & Disagree & Agree \\
\hline I usually read the instruction label before I take the medication & $116(25.3 \%)$ & $342(74.7 \%)$ \\
\hline Do you follow the directions on the label? & $116(25.3 \%)$ & $342(74.6 \%)$ \\
\hline Do you tell the pharmacist about concurrent medication at the time of OTC purchase & $112(25.1 \%)$ & $334(74.6 \%)$ \\
\hline Do you have home pharmacy? & $47(10.3 \%)$ & $411(89.7 \%)$ \\
\hline Do you check expiry date of drug? & $33(7.2 \%)$ & $424(92.8 \%)$ \\
\hline During 1 year, How many times did you visit the pharmacy? & $\begin{array}{l}<=2 \text { times: } \\
229(52.4 \%)\end{array}$ & $\begin{array}{l}>2 \text { time: } \\
208(47.6 \%)\end{array}$ \\
\hline What do you do if the drug doesn't improve your medical symptoms & $\begin{array}{l}\text { Increase dose } \\
\text { Decrease dos } \\
\text { Return to pha } \\
\text { Go to physici } \\
\text { Increase dura }\end{array}$ & \\
\hline
\end{tabular}

Table 4. Patient Physician and Pharmacist relationship

\begin{tabular}{lll}
\hline Patient Physician Relationship & Agree & Disagree \\
\hline Have you consulted a pharmacist in relationship with your current symptoms? & $107(23.4 \%)$ & $47(10.3 \%)$ \\
\hline Do you inform the physician about the consumption of this drug? & $356(77.6 \%)$ & $103(22.3 \%)$ \\
\hline Does the Pharmacist or physician explain the severity of your disease? & $336(73.2 \%)$ & $123(26.8 \%)$ \\
\hline The pharmacist or the physician give reliable advice about drugs? & $397(86.5 \%)$ & $62(13.5 \%)$ \\
\hline Do you understand how to take the medications? & $440(95.9 \%)$ & $19(4.1 \%)$ \\
\hline I would be comfortable receiving drugs directly from a pharmacist? & $282(61.6 \%)$ & $176(38.4 \%)$ \\
\hline
\end{tabular}

Most participants reported returning to the pharmacy if the symptoms didn't improve 178 (39.2\%). 27\% reported retuning to physicians, $13.3 \%$ increased the duration of consumption, $11.4 \%$ increased the dose consumed, and $7.3 \%$ reported reducing the dose. Older participants reported more returning to physicians than younger participants. (Table 3)

Many participants consulted physician or pharmacists for their current symptoms 410 (89.7\%). 356 (7.6\%) informed their physicians about the consumption of this drug. 336 (73.2\%) of the pharmacists explained the severity of their diseases. $397(86.5 \%)$ of the pharmacists give reliable advice about the drug. 440 (95.9\%) understood how to take their medications and $282(61.6 \%)$ were comfortable to receive their drug directly from the pharmacies without prescription. (Table 4)

\section{Discussion}

Overall this study revealed the knowledge and attitude of general public toward OTC use in Lebanon. Selfpresentation on previous experience of the same medication or through pharmacist advice. Almost half requested OTC medications more than twice per year. This show the important role of pharmacist in guiding the patients on the correct OTC use. Around 60\% agreed that some non-prescription medicines may cause dependency or addiction if taken for a long period of time.

A large nationally representative survey in the US revealed high levels of concurrent prescription and nonprescription drug use in respondents, leading to concerns about unintended interactions; [14] this confirmed similar findings reported by Finnish researchers who utilized data from a population based interview survey on health care. [15] Doctors and pharmacists must be aware of polypharmacy and any non-prescribed remedies that the patient may be taking. [16] This is reassuring especially with increasing availability of potent medications without prescription and the increased potential for interactions. $[17,18]$

In the present study $25.3 \%$ didn't read the instructions on the label. This proportion increased after adding those who reporting rarely read the instructions to $56.5 \%$. they would be at risk of misusing OTC medicines. This percentage was less than that reported in other studies as Everyday Healthcare Study (96.0\% read the instructions). [19] This decrease could be due to an increase in people's confidence over time in relation to self-treatment. It could also be due to people's belief that only safe medicines are permitted to be sold without prescription and that OTC medicines do not usually have serious side-effects. [3] In 1999, The Food and Drug Administration (FDA) issued a regulation regarding new standardized easy-to-understand labels for OTC products that all such products in the USA will be required to adopt by 2005 [20].

The most common reason reported for self-medication was prior experience of the same medication similarly to that reported in other studies [12,21-26]. The uncontrolled consumption and monitoring of consumption of medicines in Lebanon is one reason of confusion among medications. Most patients reported also keeping these medications at home for future use. These factors increase the self-medication use.

Research commissioned by the Proprietary Association of Great Britain (PAGB) in 1997 has revealed GP support for self-medication is growing, with $83 \%$ of the GPs stating that they would feel comfortable about referring patients to pharmacists. [19] Pharmacists (without a doctor's prescription) were among the top 5 sources of 
obtaining these medications in this survey. In Lebanon, there are about 1923 registered community pharmacies, each serving an average of 2000 people. [27] Since 2003, stricter measures have been taken to prevent any drug sales without a doctor's prescription, but the implementation of the laws remains questionable. If regulations were truly being enforced, pharmacists (without a prescription) would not have arisen as one of the leading sources of medication.

Doctors, as shown in this study, may be potentially contributing to the increased availability of and accessibility to these psychoactive prescription medications. While the mechanisms remain unclear, it is possible that physicians who are family members or family friends may be an outlet. In a country where the health care system is not centralized, one physician may also be unaware of another's prescription note, potentially leading to multiple simultaneous prescriptions. Diversion is also a potential source for misuse; $20 \%$ of the lifetime medical users in this study diverted their medication, similar to other settings $(22.9 \%)$. [28,29]

This study was the first prospective study done in Lebanese population estimating the knowledge toward self-medication use and abuse of OTC products. However, our study suffers from several limitations. To begin with, since not all pharmacies accepted to participate to the study, the sample may not be representative of Lebanese population. Second, there could also be a possibility of respondent and information bias, since the results of our study are based on a face to face questionnaire. This study suffered from most consumer-based surveys issues, mostly the willingness or not of subjects coming to the pharmacy to spend tie filling a questionnaire or speaking to an interviewer.

\section{Conclusion}

The findings of this research should form the basis for future interventional plans to maximize benefits and minimize risks of self-medication practice. To achieve this, authors suggest expanding educational programs to population, pharmacists and health care providers should be implemented to limit the potential misuse/abuse of these medicines. Additionally, the health authorities have to implement their regulations to prohibit the selling of POM. Considering the breadth of medicine available without a prescription and the problems that can arise with medication use, community pharmacies in Lebanon have the potential to make a huge impact in ensuring medicines are used appropriately.

\section{Conflicts of Interest}

The authors declare no conflicts of interest.

\section{Acknowledgements}

The authors thank the Lebanese University for funding this project.

\section{References}

[1] Hardon A, Sjaak VdG. Hazards of self-medication. World Health Forum. 1987; 8: 469-71.

[2] Bradley C, Bond C. Increasing the number of drugs available over the counter: arguments for and against. . Br J Gen Pract 1996; 46: 121-2.

[3] Hughes L, Whittlesea C, Luscombe D. Patients' knowledge and perceptions of the side-effects of OTC medication. J Clin Pharm Ther. 2002; 27: 243-8.

[4] Hughes GF, McElnay JC, Hughes CM, McKenna P. Abuse/misuse of non-prescription drugs. Pharm World Sci. 1999; 21: 251-5.

[5] Robinson GM, Robinson S, McCarthy P, Cameron C. Misuse of over-the-counter codeine-containing analgesics: dependence and other adverse effects. N Z Med J. 2010; 123: 59-64.

[6] Reed K, Bond A, Witton J, Cornish R, Hickman M, Strang J. The changing use of prescribed benzodiazepines and z-drugs and of over-the-counter codeine-containing products in England: a structured review of published English and international evidence and available data to inform consideration of the extent of dependence and harm. London and Bristol: The National Addiction Centre, Kings College London and School of Social and Community Medicine, University of Bristol, United Kingdom. 2011.

[7] Agyapong CM, Sarvage M, Sighn K, Farren C, McLoughlin D. Use of codeine-containing medicines by patients admitted to an acute psychiatric hospital. European Psychiatry. 2011; 26: 1.

[8] Marie Claire Van Hout. A Respectable Addiction: Over The Counter Codeine Use, isuse and Dependence. 2014. p. 107-18.

[9] EMCDDA Annual Report. Annual report on the state of the drugs problem in Europe, Lisbon, Portugal: European Monitoring Centre of Drugs and Drug Addiction. 2011.

[10] Frei MY, Nielsen S, Dobbin MD, Tobin CL. Serious morbidity associated with misuse of over-the-counter codeine-ibuprofen analgesics: a series of 27 cases. Med J Aust. 2010; 193: 294-6.

[11] Cooper RJ. Over-the-counter medicine abuse - a review of the literature. J Subst Use. 2013; 18: 82-107.

[12] Yousef AM, Al-Bakri AG, Bustanji Y, Wazaify M. Selfmedication patterns in Amman, Jordan. Pharm World Sci. 2008; 30: 24-30.

[13] Wazaify M, Shields E, Hughes CM, McElnay JC. Societal perspectives on over-the-counter (OTC) medicines. Fam Pract. 2005; 22: 170-6.

[14] Kaufman DW, Kelly JP, Rosenberg L, Anderson TE, Mitchell AA. Recent patterns of medication use in the ambulatory adult population of the United States: the Slone survey. JAMA. 2002; 287: 337-44.

[15] Sihvo S, Klaukka T, Martikainen J, Hemminki E. Frequency of daily over-the-counter drug use and potential clinically significant over-the-counter-prescription drug interactions in the Finnish adult population. Eur J Clin Pharmacol. 2000; 56: 495-9.

[16] Honig PK, Cantilena LR. Polypharmacy. Pharmacokinetic perspectives. Clin Pharmacokinet. 1994; 26: 85-90.

[17] Honig PK, Gillespie BK. Drug interactions between prescribed and over-the-counter medication. Drug Saf. 1995; 13: 296-303.

[18] Bell H, McElnay J, Hughes CM. Societal perspectives on the role of the community pharmacist and community-based pharmaceutical services. . J Soc Adm Pharm. 2000; 17: 119-28.

[19] British Market Research Bureau (BMRB). Everyday healthcare study: a consumer study of self-medication in Great Britain. London: Proprietary Association of Great Britain. 1997.

[20] Nightingale S. New easy-to-understand labels for OTC drugs. J Am Med Assoc. 1999; 281: 113.

[21] Jalilian F, Hazavehei SM, Vahidinia AA, Jalilian M, Moghimbeigi A. Prevalence and related factors for choosing self-medication among pharmacies visitors based on health belief model in Hamadan Province, west of Iran. J Res Health Sci. 2013; 13: 81-5.

[22] Al-Azzam SI, Al-Husein BA, Alzoubi F, Masadeh MM, Al-Horani MA. Self-medication with antibiotics in Jordanian population. Int J Occup Med Environ Health. 2007; 20: 373-80.

[23] Mumtaz Y, Jahangeer A, Mujtaba T, Zafar SN. Self Medication among University Students of Karachi. JLUMHS. 2011; 10: 102-5.

[24] James H, Handu SS, Khaja KA, Sequeira RP. Influence of medical training on self-medication by students. Int J Clin Pharmacol Ther. 2008; 46: 23-9. 
[25] Zafar SN, Syed R, Waqar S, Zubairi AJ, Vaqar T, Shaikh M, et al. Self-medication amongst university students of Karachi: prevalence, knowledge and attitudes. J Pak Med Assoc. 2008; 58: 214-7.

[26] Scicluna EA, Borg MA, Gur D, Rasslan O, Taher I, Redjeb SB, et al. Self-medication with antibiotics in the ambulatory care setting within the Euro-Mediterranean region; results from the ARMed project. J Infect Public Health. 2009; 2: 189-97.
[27] World Health Organization. Measuring Transparency to Improve Good Governance in the Public Pharmaceutical Sector. 2007.

[28] Haydon E, Rehm J, Fischer B, Monga N, Adlaf E. Prescription drug abuse in Canada and the diversion of prescription drugs into the illicit drug market. Can J Public Health. 2005; 96: 459-61.

[29] Goldsworthy RC, Schwartz NC, Mayhorn CB. Beyond abuse and exposure: framing the impact of prescription-medication sharing. Am J Public Health. 2008; 98: 1115-21. 